\title{
A GENERALIZATION OF THE HELLY SELECTION THEOREM
}

\author{
BY KEITH SCHRADER
}

Communicated by Victor Klee, November 16, 1971

1. Introduction. In this paper we consider sequences $\left\{y_{k}\right\}$ of real valued functions defined on an interval $I$. We are interested in finding conditions which when satisfied by the sequence $\left\{y_{k}\right\}$ guarantee the existence of a subsequence of $\left\{y_{k}\right\}$ which converges pointwise on $I$. With this in mind we make the following definition.

DEFINITION 1.1. Let $f: I \rightarrow R$ and consider the set $\mathscr{P}$ of all finite nonempty partitions $P=\left\{x_{1}, x_{2}, \ldots, x_{n}\right\}$ of $I$ where $n \geqq 1$ and $x_{1}<x_{2}$ $<\cdots<x_{n}$. We denote by $T(f)$ the oscillation of $f$ on $I$ and define it by

$$
\begin{aligned}
T(f)=\sup _{P \in \mathscr{P}}\left\{\sum_{i=1}^{n}\left|f\left(x_{i}\right)\right|:(-1)^{i} f\left(x_{i}\right)>0 \forall i\right. \\
\\
\left.\quad \text { or }(-1)^{i} f\left(x_{i}\right)<0 \forall i \text { or }(-1)^{i} f\left(x_{i}\right)=0 \forall i\right\} .
\end{aligned}
$$

For a function $f$ which is nonnegative on $I$ the oscillation of $f$ on $I, T(f)$, is the supremum of $f$ on $I$. It is not the case, however, that the set of $f$ for which $T(f)$ is finite forms a Banach space with norm $T(f)$ since closure under addition is not satisfied. It is also not the case that the set of $f$ for which $T(f)$ is finite forms a metric space with metric given by $d(f, g)$ $=T(f-g)$ because the triangle inequality is not satisfied.

Our main result, for which we give a number of applications later, is the following.

THEOREM 1.2. Let $\left\{y_{k}\right\}$ be such that $y_{k}: I \rightarrow R$. If $T\left(y_{k}-y_{j}\right) \leqq M$ for all $k, j$ then $\left\{y_{k}\right\}$ contains a subsequence which converges pointwise on $I$.

The original motivation for this theorem comes from the study of boundary value problems. In [3] the author showed, among other things, that if $\left\{y_{k}\right\}$ is a uniformly bounded sequence of continuous real valued functions defined on an interval $I$ having the property that there exists a positive integer $N$ such that $y_{k}$ and $y_{j}$ are not equal at more than $N$ values of $x$ for $k \neq j$ then $y_{k}$ contains a subsequence which converges at every point in $I$. This result is a corollary of Theorem 1.2. A more complete description of the connection between such convergence theorems and the

AMS 1969 subject classifications. Primary 4020; Secondary 3436.

Key words and phrases. Sequences of functions, pointwise convergence.

Copyright $(\mathcal{O})$ American Mathematical Society 1972 
study of boundary value problems may be found in [3] along with a list of references.

2. Preliminary results. We begin by stating a known result due to Ramsey which we will refer to repeatedly. This result and its proof may be found in [1, Theorem A] or [2, Theorem A, p. 82].

THEOREM 2.1. Let $\Gamma$ be an infinite class, $u$ and $r$ positive integers; and let all those subclasses of $\Gamma$ which have exactly $r$ members, or, as we may say, let all r-combinations of the members of $\Gamma$ be divided in any manner into $u$ mutually exclusive classes $C_{i}(i=1,2, \ldots, u)$, so that every $r$-combination is a member of one and only one $C_{i}$. Then, assuming the Axiom of Selections, $\Gamma$ must contain an infinite subclass $\Delta$ such that all the r-combinations of the members of $\Delta$ belong to the same $C_{i}$.

COROLlaRY 2.2. If $\left\{f_{k}\right\}$ is any sequence of real valued functions defined on an interval $J$ then either there is a subsequence $\left\{h_{j}\right\}$ of $\left\{f_{k}\right\}$ such that $\left\{h_{j}\right\}$ is a monotone sequence on $J$ or else there is a subsequence $\left\{h_{j}\right\}$ of $\left\{f_{k}\right\}$ such that if $i \neq j$ there are $t, \tau \in J$ depending on $i, j$ with $h_{i}(t)>h_{j}(t)$ and $h_{i}(\tau)$ $<h_{j}(\tau)$.

PROOF. If there are only finitely many functions in $\left\{f_{k}\right\}$ which are distinct on $J$ then infinitely many are identical on $J$ and we are done. Thus we may assume there are infinitely many functions in $\left\{f_{k}\right\}$ which are distinct on $J$ and, by picking a subsequence if necessary, we may assume all the $f_{k}$ are distinct. Let $u=r=2$ and $\Gamma=\left\{f_{k}\right\}$. Let $C_{1}=\left\{\left\{f_{k}, f_{j}\right\}: k \neq j, f_{k}(t)\right.$ $\leqq f_{j}(t) \quad \forall t \in J$ or $\left.f_{k}(t) \geqq f_{j}(t) \quad \forall t \in J\right\}$ and $C_{2}=\left\{\left\{f_{k}, f_{j}\right\}: k \neq j, f_{k}(t)\right.$ $>f_{j}(t)$ for some $t \in J$ and $f_{k}(\tau)<f_{j}(\tau)$ for some $\left.\tau \in J\right\}$. The result now follows from Theorem 2.1 .

COROLLARY 2.3. If $\left\{f_{k}\right\}$ is any sequence of real valued functions defined on an interval $J$ and $\varepsilon>0$ is any number then either there is a subsequence $\left\{h_{j}\right\}$ of $\left\{f_{k}\right\}$ such that, for $i \neq j,\left|h_{j}(t)-h_{i}(t)\right|<\varepsilon$ for all $t \in J$ or else there is a subsequence $\left\{h_{j}\right\}$ of $\left\{f_{k}\right\}$ such that, for $i \neq j,\left|h_{j}(t)-h_{i}(t)\right| \geqq \varepsilon$ for some $t \in J$ depending on $i, j$.

Proof. As in the proof of Corollary 2.2 we may assume the $f_{k}$ are all distinct on $J$ or we are done. Let $\Gamma=\left\{f_{k}\right\}, u=r=2, C_{1}=\left\{\left\{f_{k}, f_{j}\right\}\right.$ : $\left.k \neq j,\left|f_{k}(t)-f_{j}(t)\right|<\varepsilon \quad \forall t \in J\right\}, C_{2}=\left\{\left\{f_{k}, f_{j}\right\}: k \neq j,\left|f_{k}(t)-f_{j}(t)\right| \geqq \varepsilon\right.$ for some $t \in J\}$. The result now follows from Theorem 2.1 .

3. Proof of Theorem 1.2. For $x \in I$ we have $\left|y_{k}(x)\right| \leqq\left|y_{k}(x)-y_{1}(x)\right|$ $+\left|y_{1}(x)\right| \leqq M+\left|y_{1}(x)\right|$ so the sequence $\left\{y_{k}\right\}$ is pointwise bounded and hence any monotone subsequence of $\left\{y_{k}\right\}$ is convergent.

Let $\left\{J_{i}\right\}$ be an enumeration of all the nonempty open intervals contained in $I$ with rational endpoints. 
It follows from Corollary 2.2 applied to $J_{1}$ that either there is a subsequence of $\left\{y_{k}\right\}$, again denoted by $\left\{y_{k}\right\}$, which is monotone and convergent on $J_{1}$ or else there is a subsequence of $\left\{y_{k}\right\}$, again denoted by $\left\{y_{k}\right\}$, such that, for $k \neq j, y_{k}(t)>y_{j}(t)$ and $y_{k}(\tau)<y_{j}(\tau)$ for some $t, \tau \in J_{1}$. We now repeat the process described in the previous sentence consecutively on the intervals $J_{2}, J_{3}, \ldots$ and then take the diagonal subsequence, denoted by $\left\{y_{k}\right\}$ again. This sequence has the property that on any of the intervals $\boldsymbol{J}_{i}$ it is either monotone on $J_{i}$ for $k$ sufficiently large and hence converges on $J_{i}$ or else for every $k \neq j$ sufficiently large there are $t, \tau \in J_{i}$ such that $y_{k}(t)$ $>y_{j}(t)$ and $y_{k}(\tau)<y_{j}(\tau)$.

Now using $J_{1}$ and $\varepsilon=1$ it follows from Corollary 2.3 that either there is a subsequence of $\left\{y_{k}\right\}$, again denoted by $\left\{y_{k}\right\}$, such that, for $k \neq j, \mid y_{k}(t)$ $-y_{j}(t) \mid<\varepsilon=1$ for all $t \in J_{1}$ or else there is a subsequence of $\left\{y_{k}\right\}$, again denoted by $\left\{y_{k}\right\}$, such that if $k \neq j$ there is some $t \in J_{1}$ for which $\mid y_{k}(t)$ $-y_{j}(t) \mid \geqq \varepsilon=1$. We now repeat this process consecutively on $J_{1}$ using $\varepsilon=1 / 2, \varepsilon=1 / 3, \ldots, \varepsilon=1 / n, \ldots$ and then take the diagonal subsequence, again denoted by $\left\{y_{k}\right\}$. This sequence has the property that for $J_{1}$ and $\varepsilon=1 / n$ either for all $k \neq j$ sufficiently large $\left|y_{k}(t)-y_{j}(t)\right|<\varepsilon=1 / n$ holds for all $t \in J_{1}$ or else for all $k \neq j$ sufficiently large there is some $t \in J_{1}$ with $\left|y_{k}(t)-y_{j}(t)\right| \geqq \varepsilon=1 / n$.

We now repeat the entire process described in the preceding paragraph consecutively on the intervals $J_{2}, J_{3}, \ldots$ and then take the diagonal subsequence, again denoted by $\left\{y_{k}\right\}$. This sequence has the property that for $J_{i}$ and $\varepsilon=1 / n$ then either for every $k \neq j$ sufficiently large, depending on $i$ and $n,\left|y_{k}(t)-y_{j}(t)\right|<\varepsilon=1 / n \quad \forall t \in J_{i}$ or else for every $k \neq j$ sufficiently large, depending on $i$ and $n,\left|y_{k}(t)-y_{j}(t)\right| \geqq \varepsilon=1 / n$ for some $t \in J_{i}$, depending on $k, j$.

We will now show using proof by contradiction that $\left\{y_{k}\right\}$ converges at all but countably many values of $x \in I$. For $x \in I$ such that $\left\{y_{k}(x)\right\}$ is not convergent let $\left\{F_{x i}\right\}$ be the subsequence of $\left\{J_{i}\right\}$ consisting of the intervals which contain $x$. There must be a smallest positive integer $n_{x i}$ such that $\left|y_{k}(t)-y_{j}(t)\right| \geqq \varepsilon=1 / n_{x i}$ for all $k \neq j$ sufficiently large for some $t \in F_{x i}$ or else $\left\{y_{k}\right\}$ is Cauchy on $F_{x i}$ and hence converges at $x$ which would contradict the choice of $x$. If $\varlimsup_{\lim _{i \rightarrow+\infty}} n_{x i}=+\infty$ then there is a subsequence $n_{x i(\alpha)}$ of $\left\{n_{x i}\right\}$ such that $\lim _{\alpha \rightarrow+\infty} n_{x i(\alpha)}=+\infty$ and by the definition of $n_{x i(\alpha)}$ we have $\left|y_{k}(t)-y_{j}(t)\right|<1 /\left(n_{x i(\alpha)}-1\right)$ for all $k \neq j$ sufficiently large and all $t \in F_{x i(\alpha)}$. This implies $\left\{y_{k}(x)\right\}$ is Cauchy which contradicts the choice of $x$, so $\overline{\lim }_{i \rightarrow+\infty} n_{x i}=c_{x}<+\infty$. Let $d_{x}>c_{x}$ be an upper bound for the set $\left\{n_{x i}\right\}$.

If there are uncountably many $x$ at which $y_{k}(x)$ is not convergent then there is some number $d$ so that $d_{x} \leqq d$ holds for uncountably many $x$ at which $\left\{y_{k}(x)\right\}$ does not converge. Denote this uncountable set of $x$ 's by $A$.

Choose $N$ so that $N(1 / d)>M$. Now choose $u(1) \in A \cap I^{0}$ and 
$F_{u(1) i(1)} \in\left\{F_{u(1) i}\right\}$ such that $\left(I-F_{u(1) i(1)}\right) \cap A$ is uncountable. Choose $u(2) \in\left(I-F_{u(1) i(1)}\right) \cap\left(A \cap I^{0}\right)$ and $F_{u(2) i(2)} \in\left\{F_{u(2) i}\right\}$ such that $F_{u(1) i(1)}$ $\cap F_{u(2) i(2)}=\varnothing$ and $\left(I-\left(F_{u(1) i(1)} \cup F_{u(2) i(2)}\right) \cap A\right.$ is uncountable. Continuing in this way we get $\{u(1), u(2), \ldots, u(2 N+1)\}$ in $A \cap I^{0}$ and $\left\{F_{u(1) i(1)}, F_{u(2) i(2)}, \ldots, F_{u(2 N+1) i(2 N+1)}\right\}$ which are mutually disjoint. By renaming the points $u(i)$ we may assume $u(1)<u(2)<\cdots<u(2 N+1)$. Now we choose $k \neq j$ fixed but sufficiently large that for each odd positive integer $\alpha, 1 \leqq \alpha \leqq 2 N+1,\left|y_{k}(x)-y_{j}(x)\right| \geqq \varepsilon=1 / n_{u(\alpha) i(\alpha)}$ holds for some $x(\alpha) \in F_{u(\alpha) i(\alpha)}$ and for each even positive integer $\alpha, 2 \leqq \alpha \leqq 2 N, y_{k}\left(t_{\alpha}\right)$ $>y_{j}\left(t_{\alpha}\right)$ holds for some $t_{\alpha} \in F_{u(\alpha) i(\alpha)}$ and $y_{k}\left(\tau_{\alpha}\right)<y_{j}\left(\tau_{\alpha}\right)$ holds for some $\tau_{x} \in F_{u(\alpha) i(\alpha)}$. Consider the partition $P_{0}=\left\{\beta_{1}, \beta_{2}, \ldots, \beta_{n}\right\}$ where $\beta_{\alpha}=x(\alpha)$ if $\alpha$ is odd; $\beta_{\alpha}$ is omitted from $P_{0}$ if $\alpha$ is even and $y_{k}(x(\alpha-1))-y_{j}(x(\alpha-1))$ and $y_{k}(x(\alpha+1))-y_{j}(x(\alpha+1))$ have opposite signs; $\beta_{\alpha}$ is taken to be $t_{\alpha}$ if $y_{k}(x(\alpha-1))-y_{j}(x(\alpha-1))<0$ and $y_{k}(x(\alpha+1))-y_{j}(x(\alpha+1))<0 ; \beta_{\alpha}$ is taken to be $\tau_{\alpha}$ if $y_{k}(x(\alpha-1))-y_{j}(x(\alpha-1))>0$ and $y_{k}(x(\alpha+1))$ $-y_{j}(x(\alpha+1))>0$. We observe this implies that $T\left(y_{k}-y_{j}\right) \geqq N(1 / d)>M$ for this fixed $k \neq j$ which contradicts the hypotheses of the theorem.

We conclude that $\left\{y_{k}\right\}$ converges for all but countably many values of $x$ and hence a subsequence can be chosen which converges at every point.

COROLLARY 3.1. Let $\left\{y_{k}\right\}$ be a sequence of real valued functions defined on a nonempty set $S \subset R$. If $T\left(y_{k}-y_{j}\right) \leqq M$ for all $k, j$ (where partition points are taken in $S$ instead of I) then $\left\{y_{k}\right\}$ contains a subsequence which converges pointwise on $S$.

Proof. Define $\left\{z_{k}\right\}, z_{k}: R \rightarrow R$ by

$$
\begin{aligned}
z_{k}(x) & =y_{k}(x), & & x \in S, \\
& =0, & & x \notin S,
\end{aligned}
$$

then $T\left(z_{k}-z_{j}\right)=T\left(y_{k}-y_{j}\right) \leqq M$ so Theorem 1.2 applies.

COROLlary 3.2 (Helly Selection Theorem). Let $\left\{y_{k}\right\}$ be a sequence of functions of bounded variation with $y_{k}:[a, b] \rightarrow R$ and let there exist a number $K$ such that $\left|y_{k}(x)\right| \leqq K$ for $k=1,2, \ldots$ and $x \in[a, b]$ and such that $V_{a}^{b}\left(y_{k}\right) \leqq K$ for $k=1,2, \ldots$ where $V_{a}^{b}\left(y_{k}\right)$ is the variation of $\left\{y_{k}\right\}$ on $[a, b]$. Then there exists a subsequence of $\left\{y_{k}\right\}$ which converges at every point.

Proof. $T\left(y_{k}-y_{j}\right) \leqq 2 K+V_{a}^{b}\left(y_{k}-y_{j}\right) \leqq 2 K+V_{a}^{b}\left(y_{k}\right)+V_{a}^{b}\left(y_{j}\right) \leqq 4 K$. In fact, the limit function is of bounded variation but we are not concerned with that here.

Corollary 3.3. Let $\left\{y_{k}\right\} \in C(I)$ and $D_{k j}=\left\{x: x \in I,\left|y_{k}(x)-y_{j}(x)\right|>0\right\}$. Then we know that $D_{k j}=\bigcup_{n=1}^{+\infty} I_{k j n}$ where each $I_{k j n}$ is a relatively open interval in $I$ and $I_{k j n} \cap I_{k j m}=\varnothing$ for $n \neq m$. If $\sum_{n=1}^{+\infty} \sup _{x \in I_{k j n}}\left|y_{k}(x)-y_{j}(x)\right|$ 
$\leqq M$ for all $k, j$ then $\left\{y_{k}\right\}$ contains a subsequence which converges at each point of $I$.

COROllary 3.4. Let $y_{k} \in C(I)$ and $K$ a fixed positive integer such that $\left\{x: x \in I,\left|y_{k}(x)-y_{j}(x)\right|>0\right\}$ contains no more than $K$ components for each $k, j$. If $\left|y_{k}(x)\right| \leqq H$ holds, for $x \in I$ and $k=1,2, \ldots$, then $\left\{y_{k}\right\}$ contains $a$ subsequence which converges at every point of $I$.

ProOF. $T\left(y_{k}-y_{j}\right) \leqq K H$.

\section{REFERENCES}

1. F. Ramsey, On a problem of formal logic, Proc. London Math. Soc. (2) 30 (1930), 264-286.

2. - The foundations of mathematics, Harcourt Brace, New York, 1931.

3. K. Schrader, A pointwise convergence theorem for sequences of continuous functions, Trans. Amer. Math. Soc. 159 (1971), 155-163.

Department of Mathematics, University of Missouri, Columbia, Missouri 65201 